\title{
The Flow of Information from Nucleus to Golgi Is Contingent upon Nuclear Membrane Synthesis and Protraction of the Ceramide-Containing Membrane to Endoplasmic Reticulum
}

\author{
Amalia Slomiany, Bronislaw L. Slomiany \\ School of Dental Medicine, Rutgers Biomedical and Health Sciences, Newark, USA \\ Email: slomiaam@sdm.rutgers.edu
}

How to cite this paper: Slomiany, A. and Slomiany, B.L. (2018) The Flow of Information from Nucleus to Golgi Is Contingent upon Nuclear Membrane Synthesis and Protraction of the Ceramide-Containing Membrane to Endoplasmic Reticulum. Advances in Biological Chemistry, 8, 47-68.

https://doi.org/10.4236/abc.2018.83005

Received: April 25, 2018

Accepted: June 4, 2018

Published: June 7, 2018

Copyright $\odot 2018$ by authors and Scientific Research Publishing Inc. This work is licensed under the Creative Commons Attribution International License (CC BY 4.0).

http://creativecommons.org/licenses/by/4.0/

\begin{abstract}
The nucleus-initiated augmentation of ER membrane is reflected in a coordinated synthesis and intercalation of the explicit proteins and lipids required for the replacement, repair and function of the cell and its organelles. The direct connection between nucleus and the membranes containing labeled sphingosine $(\mathrm{SphN})$ and ceramide (Cer) was affirmed by determining synthetic activity of serine palmitoyltransferase (SPT). The SPT and the newly synthesized serine-labeled lipid products were identified in the Outer- and Inner-Nuclear Membrane (ONM, INM) and ER. The pulse-chase experiments disclosed that the incorporation of radiolabeled lipids into both nuclear membranes declined upon their simultaneous increase in Endoplasmic Reticulum (ER). These results, and prior findings regarding metabolic transfer of nuclear membrane phosphoinositides to the outer leaflet of ER [Slomiany and Slomiany, Health, 2011, 3, 187-199], allowed us to reason that INM and ONM are not distinct entities, but uninterrupted continuum facing nucleosol and then cytosol when protracted into segment known as ER. Consequently, the identification of SPT and its products in the inner leaflet of nuclear and ER microsomes lent credence to the luminal presence of Cer in Golgi, luminal synthesis of glycosphingolipids (GSphLs), sphingomyelin (SM), and their delivery to the outer leaflet of apical and basolateral cell membrane, respectively. The findings presented in this communication provide further support to our concept that the factual intercalation of proteins and lipids into the cell membranes can only take place during their simultaneous synthesis that is guided by the nuclear and cytosolic processes enacted in nuclear-ER membrane con-
\end{abstract}


tinuum. At the nuclear stage, the signal-specific genes expression promotes active synthesis and intercalation of lipids into the organelles' customized membrane that is protracted and articulated in ER in form of transport vesicles.

\section{Keywords}

Nucleus Membrane Biogenesis, Golgi Secretory Pathway, Serine Palmitoyl

Transferase, Ceramides Synthesis in Nuclear Membrane,

Organelle-Customized ER Transport Vesicles

\section{Introduction}

The nuclear envelope is viewed as a double bilayer membrane barrier, the entity separating and yet fully linking nuclear and cytoplasmic processes that promote and impact cellular physiological functions and organelles' and cell's restitution [1] [2] [3]. The knowledge regarding this phenomenon is extensive but its main focus on proteins and molecular transport crisscrossing envelope via nuclear pores generates incomplete and unfounded portrayal of the event [4] [5] [6]. The highly detailed pictures corroborated with labeled, nucleus specific transitory substances, fail to recognize the properties, the contribution, and the dynamic role of the membrane lipid bilayer in the pronouncement of the nuclear and cytosolic signals [7] [8] [9]. In the presently accepted interpretation, the segments referred to as Inner Nuclear Membrane (INM) are physically separated from cytosol-impacted membrane biogenesis, and thus appear frozen in the interaction with the same sets of nuclear elements. Hence, as so far envisioned, the process designed on the detailed information about segments of nuclear barrier, the complexity of genome and protein organization, the nuclear anchorage, the interaction with cytoskeleton and more, still create incomplete, metabolically inactive and lipids-deficient membrane complex [10] [11] [12] [13]. The entire anabolic process that requires trafficking of active and incessantly changing components to enter and evoke signal-specific transcription in nucleus and exit to cytosol to complete the task of translation is not documented and viable [14] [15] [16]. The fundamental vital energetic purpose of the system with the intentional and crucial function of the inner and outer nuclear membrane and their coded differences in composition subsequently reflected in ER processes is not considered. Consequently, the common orthodox view developed decades ago in which cell membrane lipids and their site-specific functions were reduced to static interaction with proteins continues to rule [17]-[22]. Likewise, the 3-dimentional computerized models of proteins shown to interact with any cocktail of the admixed lipids, further popularize lingering concept that only protein structure is of utmost significance [23] [24] [25]. Thus, the advance in the understanding of lipids involvement in the specific compartmentalization of processes remains status quo, and the lipids somatic properties, unlike their 
protein or nucleic acid counterparts, are not truly investigated. In the interim, the investigations of artificial lipid and protein composites produce semblances which further depart from the in situ dynamic specificity and the order dictated by anabolic complexes [22] [23] [24]. Irrefutably, the scheme of nuclear membrane function in the assembly of cell structures, without parallel emphasis on lipid involvement in the completion of the signal-induced processes remains incomplete, dimensionless and static.

Based on the organelle-specific lipid synthesis-derived concept, we depart from commonly accepted view of membrane proteins organization determined by proteins alone, and the existence of double nuclear barrier membrane [24] [25]. We explore further the active assembly of single bilayer nuclear envelope that generates changes in membrane exposure to nuclear components by signal-driven synthesis and restitution of the nuclear and ER lipid and protein membrane components [26] [27]. In our perception of the process, the movement across nuclear surface is achieved by specific lipid and protein synthesis in the inner and outer membrane leaflet and that is regulated by signal-encrypted expression of genes. Henceforward, we advance an idea that signal-controlled synchronized synthesis of lipids and proteins assemblies coordinately intercalate into the existing nuclear barrier, then the nucleus-determined membrane prototype exits to cytosol-exposed ER that completes orderly formation of the organelle and cell specific membranes [27] [28] [29]. This tenet of membrane biogenesis cannot be substituted or mimicked by an in vitro prepared lipid and protein assortments [23] [24], the process that never could replicate the precision of the in vivo step by step signal-controlled vesicular membrane assemblies delegated to Golgi and mitochondria transport pathways [28] [29] [30].

The dynamic process of the interaction between nuclear compartment signals and formation of code-dictated ER membrane required for the formation of transport vesicles dedicated for the specific organelle-and/or-cell membrane restitution and fulfillment of the cell assigned function, must entail simultaneous translation and synthesis of signal-specific membrane integral proteins and lipids. By the same principle, the membrane events that are initiated in the sole of nucleus are not isolated from ER but constitute unique dynamic membrane continuum that evolves and produces signal-tailored ER membrane [31]. Henceforth, the described course of events empowers all structural elements of the continuous membrane encompassing nucleus-ER organelle system and eliminates credence or the necessity of double nuclear membrane.

Discernibly, the double nuclear membrane just as ER membrane microsomes are the artifacts created during membrane fragmentation and scission of the nucleus from ER. The sealing of the membrane segments in close vicinity of the nucleus creates shapes of double nuclear membrane, the nuclear microsomes (NM), whereas fragmented ER seals into structures referred to as ER microsomes. The question that arises at this stage of investigation is whether ribosomes attached to the nuclear microsomes differ in composition from clusters of 
ribosomes seeking attachment to ER microsomes, and how that defines cell and the destination of the produced transport vesicles. While our study revealed that ER microsomes produce variety of transport vesicles whose lipid composition and appearance of the leaflet-lipid specificity defines their delivery to Golgi or Mitochondria [26] [27] [28] [29] [30], here we have investigated contribution of the nucleus-retained nuclear microsomes on the synthesis of the membranes referred in literature as Outer- and Inner Nuclear Membrane (ONM and INM). In our view both represent the continuum of the single lipid bilayer transiently facing nuclear contents (INM), one that egresses and is facing cytosol (ONM), and both are parts of the nucleus-ER membrane continuum. The investigative results presented in these studies on the metabolic activity of nuclear microsomes confirm synthesis-induced transfer of membrane with its specific labeled lipids into ER and further to the inner leaflet of Golgi. Together, the findings allowed us to speculate that the nuclear microsomes contribute to Golgi-directed transport, and in turn, to renewal of the cell membrane.

Consistently, our studies on membrane lipids synthesis disclose several features that decide upon dynamic linkage of cell nucleus with cell organelles and the cell vital functions. We demonstrate here the significance of active assembly of the nuclear membrane, the importance of sphingolipids synthesis in the assembly of inner leaflet of the uninterrupted membrane continuum enveloping nucleus and creating ER membrane with assets that define Golgi-directed transport and Golgi anabolic activity expressed in synthesis of GSLs and SM.

\section{Materials and Methods}

\subsection{Isolation of Cell Organelles}

In the isolation of the intact organelles the meticulous fragmentation of cell structure is crucial. The preparation of the membrane-surrounded components, prevention of their cross contamination and recovery of the membrane fragments-free cell cytosol require multitude of preparatory tasks and sacrificing either quality or quantity. Hence, to minimize the breakup of nuclei and recovery of the original cell cytosol, this investigation utilized organelles obtained from subdivided cell preparation. One part of the cells was fragmented with the emphasis on preparation of the intact nuclei and of the genuine contaminant-free cell cytosol. The other portion of the cells was subjected to the procedures described in earlier investigations in which the pure microsomes of Endoplasmic Reticulum (ER), Golgi, and the endosomes were indispensable for the optimal fusion of the transport vesicles with correct organelle [27] [28] [29] [30] [31]. Briefly, the cells were prepared from rat liver as described previously [28] [32] [33] [34] [35]. The single cells that were separated from larger debris with aid of specific cell size nylon mesh were centrifuged at $50 \times \mathrm{g}$ for $2 \mathrm{~min}$, washed twice with the enzyme-free medium, twice with the Minimum Essential Medium (MEM) and counted in hemocytometer. The cells were then incubated in MEM for 3 hours with or without radiolabel, divided into two aliquots; one was used 
for preparation of nuclei and cell cytosol while the other for ER, Golgi, endosomes and cell membranes [26] [36]. In the experiments dedicated to the determination of lipid synthesis with cell cytosol derived from untreated or RNase-treated cytosol, the preparations of nuclei, ER, Golgi, endosomes and cell membranes were additionally rinsed with phosphate buffered saline (PBS). The $2 \mathrm{M}$ urea-PBS was used in the experiments to determine the presence and the location of the shingosine-generating enzyme (SPT) in the nuclear membranes that in the outset of the studies was identified in the inner and outer leaflet of ER microsomes [29]. As determined earlier the outer leaflet $56 \mathrm{KDa}$ SPT was released from ER membranes by the treatment with urea-PBS, whereas the $58 \mathrm{KDa}$ SPT remained in the urea extracted ER microsomes. The activity of both forms of SPT was verified by assay utilizing radiolabeled $\left[{ }^{3} \mathrm{H}\right]$ serine or $\left[{ }^{3} \mathrm{H}\right]$ palmitate and thin layer chromatographic analysis of labeled products [27] [32] [33] [34] [35] [36].

The nuclear membranes, ER, and the organelles rebuilt via Golgi vesicular pathway were analyzed for the presence of radiolabeled ( $\mathrm{SphN}$ ) and Ceramides (Cer) to establish whether they originate from the synthetic activity of SPT intercalated into the nuclear and ER membrane. Consequently, the serine-radiolabeled ER-derived transport vesicles, and their fusion with Golgi was assessed by determination of the contents of labeled Cer in the vesicles and, following fusion with the organelle, its transfer to Golgi membranes. In continuation, the Golgi-generated transport vesicles were analyzed for the contents of labeled Cer, glycosphingolipids (GSLs) and sphingomyelin (SM). To establish location of SM in outer leaflet of Golgi membrane and in its vesicles, the organelle and the vesicles that remained in fusion solution after reaction with cell apical and/or basolateral membranes were treated with SMase [29]. In all experiments, the cell cytosol (CC) was used at concentration of $15 \mathrm{mg}$ protein/ml of incubation mixture and was enriched with $50 \mu \mathrm{M}$ ATP, $250 \mu \mathrm{M}$ CTP, $50 \mu \mathrm{M}$ GTP, 5 $\mathrm{mM}$ creatine phosphate, $8.0 \mathrm{IU} / \mathrm{ml}$ creatine kinase, and where indicated $25 \mu \mathrm{g} / \mathrm{ml}$ RNase, $10 \mathrm{mM}$ UDP-Glc and $10 \mu \mathrm{M}$ palmitoyl CoA [27] [32] [33] [34] [35] [36].

\subsection{Preparation of Nuclei and Transport-Active Cell Cytosol (CC)}

The viable cells, homogenized for $10 \mathrm{sec}$ at $600 \mathrm{rpm}$ in 3 volumes of buffer containing $0.25 \mathrm{M}$ sucrose; $50 \mathrm{mM}$ TRIS- $\mathrm{HCl}(\mathrm{pH} 7.4), 25 \mathrm{mM}$ magnesium acetate and $10 \mathrm{mM}$ each of aprotinin, leupeptin, chemostatin, and $1 \mathrm{mM}$ phenylmethylsulfonylfluoride, were centrifuged at 5,000 $\times \mathrm{g}$ for $15 \mathrm{~min}$. The nuclei were purified further by the resuspension of the sediment in the buffer described above until small membranous impurities were no longer detectable under microscope, and then the fraction was used for identification of the serine-labeled SphN and Cer, or first subjected to isolation of the outer- and inner nuclear membrane (ONM, INM) and then each fraction was analyzed for the contents of the labeled sphingoid lipids. The original label-free cell cytosol, the first supernatant separated from nuclei and containing remaining cell organelles was di- 
luted with 2 volumes of homogenization buffer, and centrifuged at 150,000 $\times \mathrm{g}$ for $1 \mathrm{~h}$. Thus, obtained organelle-free cell cytosol was adjusted to $15-18 \mathrm{mg}$ protein/ml, admixed with an ATP generating system consisting of $40 \mathrm{mM}$ ATP, 200 $\mathrm{mM}$ creatine phosphate, $2000 \mathrm{units} / \mathrm{ml}$ creatine phosphokinase, and referred to as transport active cell cytosol or active cytosol (CC). The first supernatant separated from experiments performed with labeled serine was used to isolate mitochondria [28].

\subsection{Preparation of Cell Organelles and Membranes}

The cell membranes and subcellular organelles (mitochondria, ER, Golgi) were recovered from cold or radiolabeled cells as described earlier [27] [28] [32] [33] [34] [35] [36]. The ER and Golgi organelles sediment, remaining after separation of nuclei, mitochondria and cell cytosol, was suspended in buffer containing 0.2 M PIPES (pH 6.9), $2 \mathrm{M}$ glycerol, $1 \mathrm{mM}$ EGTA and $1 \mathrm{mM}$ magnesium acetate and applied on the top of discontinuous gradient of $2.0 / 1.5 / 1.3 / 1.0 \mathrm{M}$ sucrose and centrifuged at $100,000 \times \mathrm{g}$ for $16 \mathrm{~h}$. The cell membranes were recovered from 1.0M sucrose, Smooth Endoplasmic Reticulum (SER) from 1.3 M sucrose, Rough Endoplasmic Reticulum (RER) from 1.5 M sucrose and Golgi from the top of the 2.0 M sucrose. Each sucrose-separated fraction was subjected to further purification. The cell membranes were washed with original PIPES buffer and centrifuged at $3000 \mathrm{rpm}$ for $2 \mathrm{~min}$. To separate cells' membrane, the buffer was adjusted with $0.2 \%$ Triton X-100 and the mixture incubated at $4^{\circ} \mathrm{C}$ for $5 \mathrm{~min}$ [27] [36]. This treatment resulted in breaking up the phosphoglycerides-rich membranes into smaller segments and thus allow us to separate cell membranes containing cholesterol, sphingolipids and glycoproteins. The latter membranes were recovered by low speed centrifugation at $3000 \mathrm{rpm}$ for $2 \mathrm{~min}$.

\subsection{Generation and Purification of Transport Vesicles}

Serine-labeled ER- and/or Golgi-derived transport vesicles were generated in the presence of cold CC [27] [29] [34] [35] [37] [38]. The ER and/or Golgi mixed with cytosol, ATP-generating system, UTP, CTP GTP, fatty acyl CoA and water soluble cold lipids precursors, were incubated for $30 \mathrm{~min}$ at $37^{\circ} \mathrm{C}$, centrifuged over $0.3 \mathrm{M}$ sucrose and treated with stripping buffer at $2^{\circ} \mathrm{C}$ for $15 \mathrm{~min}$ followed by centrifugation at $10,000 \times \mathrm{g}$ for $10 \mathrm{~min}$ to separate transport vesicles from $\mathrm{ER}$ or Golgi membranes. The separated from maternal membranes transport vesicles were recovered from the supernatant resulting from centrifugation of the supernatant mixture at $150,000 \times \mathrm{g}$ for $60 \mathrm{~min}$. The crude fraction of the transport vesicles was suspended in 55\% sucrose, overlaid with 55\% - 30\% gradient and centrifuged at $150,000 \times \mathrm{g}$ for $16 \mathrm{~h}$. The purified transport vesicles were recovered from the gradients as reported earlier [27] [32] [33] [34] [35] [36]. The same procedure was repeated with Golgi recovered after ER-derived vesicles fusion to identify the vesicles and their radiolabeled lipids in the vesicles destined to cell membranes and endosomes. At this stage, the Golgi-derived vesicles were 
reacted first with endosomes [29] and the material remaining in the solution destined to cell membrane were analyzed for the presence of SM and GSL. The fraction of ER transport vesicles destined to mitochondria was recovered from cell cytosol after ER vesicles fused with Golgi. The lipid extract from the recovered material was analyzed for the serine-labeled lipids before and after alkaline methanolysis [32].

\subsection{Fusion of ER-derived Transport Vesicles with Golgi}

One volume of ER transport vesicles (1.3 - $1.5 \mathrm{mg}$ protein/ml) was suspended in one volume of active CC (15 mg protein/ml), and added to one volume of cell organelles $(5 \mathrm{mg}$ protein $/ \mathrm{ml})$. The reaction was allowed to proceed from 0 - 30 min at $4^{\circ} \mathrm{C}$ (control) and at $37^{\circ} \mathrm{C}$ in the presence of ATP regenerating system consisting of $40 \mathrm{mM} \mathrm{ATP}, 200 \mathrm{mM}$ creatine phosphate, 2000 units $/ \mathrm{ml}$ of creatine phosphokinase, or in the ATP depleting system containing $5 \mathrm{mM}$ glucose and 500 units/ml hexokinase. After incubation, the Golgi organelles were recovered by centrifugation through three volumes of $0.5 \mathrm{M}$ sucrose at $3000 \mathrm{rpm}$ for 5 min. The remaining vesicles recovered from the supernatant after incubation with Golgi were used in the fusion experiments with mitochondria, or subjected to lipid extraction and identification of serine labeled, alkaline methanolysis susceptible phosphoglycerides and alkaline methanolysis-resistant Cer. One volume of the recovered vesicles $(0.9-1.1 \mathrm{mg} / \mathrm{ml})$ was resuspended in one volume of CC $(15 \mathrm{mg} / \mathrm{ml})$ and added to one volume of purified mitochondria $(5 \mathrm{mg} / \mathrm{ml})$ and the reaction was allowed to proceed for $30 \mathrm{~min}$ under same conditions as described for the ER vesicles fusion with Golgi. In the experiments estimating en bloc fusion of transport vesicles with Golgi and/or mitochondria, the associated but not fused vesicles were released from the membrane by subjecting the membrane fraction to treatment with $2 \mathrm{M}$ urea at $4^{\circ} \mathrm{C}$ and then the recovered organelles were centrifuged through $0.5 \mathrm{M}$ sucrose, washed and subjected to lipid analysis.

\subsection{Preparation of Outer and Inner Nuclear Membranes}

The carefully separated nuclei from cell organelles and the cell cytosol were subjected to treatments described earlier that affords mixture of membrane fragments commonly identified as outer and inner nuclear membranes (ONM, INM) [34]. To separate them into distinct fractions, the preparation of purified nuclear pellet recovered after centrifugation at 27,000 rpm in Beckman 45 Ti rotor for $1 \mathrm{~h}$ was suspended at concentration of $2 \mathrm{mg}$ protein/ml in a buffer consisting of $50 \mathrm{mM}$ TIS-HCl pH 7.4, $0.25 \mathrm{M}$ sucrose, $10 \mathrm{mM} \mathrm{MgCl}_{2}, 1 \mathrm{mM}$ DTT, $10 \mathrm{mg} / \mathrm{ml}$ leupeptin and $2 \mathrm{mM}$ PMSF and then adjusted to $1 \%$ (w/v) with sodium citrate. The preparation was then incubated on ice with gentle stirring for $30 \mathrm{~min}$, followed by centrifugation at $500 \times \mathrm{g}$ for $15 \mathrm{~min}$. The obtained supernatant contained the ONM, whereas the pellet contained INM. The INM containing fraction was subjected to DNase 1 digestion $(250 \mathrm{mg}$ DNase/ml for $14 \mathrm{~h}$ at 
$4^{\circ} \mathrm{C}$ ), and the digest separated on the gradient of $0.25-1.6-2.4 \mathrm{M}$ sucrose centrifuged at $10,000 \times \mathrm{g}$ for $2 \mathrm{~h}$. The INM were recovered from $1.6 \mathrm{M}$ sucrose boundary. The fraction of ONM, collected from citrate supernatant was subjected to the same preparative procedure as INM described above. On the average, the yield of INM to ONM was in ratio of 3:5. To remove sucrose, the separated fractions were diluted in $20 \mathrm{mM}$ phosphate buffer, $\mathrm{pH} 7.2$ and centrifuged at $17,000 \mathrm{rpm}(35,000 \times \mathrm{g})$ for one hour. Then the samples recovered from sucrose gradient were resuspended in $0.5 \mathrm{ml}$ of the same buffer and used for SPT enzyme assays and lipid analysis.

\subsection{Isolation of Serine Palmitoyltransferase (SPT) from Nuclear Microsomes (NM), Inner- and Outer-Nuclear Membrane (INM, ONM) and ER}

To determine whether SPT enzyme is present in nuclear membranes, the purified nuclei ONM, INM and ER were used for preparation of STP before and after treatment with urea as described earlier [29]. Following release of urea-extractible form, the nuclei were subjected to the procedure used to separate the Inner- and Outer Nuclear Membrane (INM, ONM) and those were used for purification of the SPT enzyme. The final purification of the enzyme was achieved by running the preparative PAGE and extraction of bands corresponding to 58 and $56 \mathrm{KDa}$ peptides. Thus purified enzymes were used in an in vitro enzyme assay [29] and in preparation of polyclonal antibodies against 58 and 56 KDa SPT. The SPT isolated from NM, INM, and ONM was subjected to immuno-blocking using antisera raised against ER-derived enzymes. The antibodies, $56 \mathrm{Ab}$ and $58 \mathrm{Ab}$ at concentration of $1.0 \mu \mathrm{g}$ were used in the assays.

\subsection{Identification of the Products Assembled by SPT of NM Membrane}

The NM membranes were subjected to lipid extraction, and high performance thin layer chromatography (HPTLC) before and after alkaline methanolysis that degraded serine-containing phosphoglycerides. Before methanolysis, the chloroform extracts equivalent to $50 \mu \mathrm{g}$ membrane proteins were chromatographed on high performance thin layer plates in solvent system consisting of chloroform/methanol/water $(65: 35: 8, \mathrm{v} / \mathrm{v} / \mathrm{v})$, and after methanolysis in chloroform/methanol/2 $\mathrm{M} \mathrm{NH}_{4} \mathrm{OH}(40: 10: 1$, v/v/v) along with $\mathrm{SphN}$ and Cer standards. After development and staining for serine-containing phosphoglycerides, SphN, and Cer, the plates was scored into 8 zones, scraped and counted.

\section{Results}

Underpinning for our hypothesis that the nuclear barrier is built of single bilayer membrane that embodies uninterrupted continuum with ER emerged during investigations of inositol phosphatides transformation culminating in synthesis of nuclear membrane phosphatidylinositol (PI) and its passage to ER [27]. In 
our understanding, the transfer of labeled PI from INM to ER, and its allocation to ER-produced transport vesicles demands an uninterrupted continuity of the membrane. To substantiate the concept that generation of the specific transport vesicles is guided by processes enacted in nucleus-ER membrane continuum, the synthesis of SphN and Cer, the markers of Golgi transport pathway, in the nuclear and ER membranes was investigated. Using radiolabeled serine as the marker of SphN and Cer assembly, the contribution of these lipids in the nuclear membrane growth and passage to ER was documented. As shown by the results presented in Figure 1, the serine incorporated into NM lipids was detected in SphN containing lipids represented by ceramides (Cer), and phosphatidylserine (PS) and their partially completed products.

The chase experiments revealed that the labeling of serine-containing lipids diminished with time in NM and increased in ER (Figure 2).

The labeled lipids increase in ER, although not exactly proportional to nuclear loss demonstrated movement of the membrane with serine-labeled lipids, including newly assembled SphN and Cer-containing and Golgi-dedicated membrane from nucleus to ER and ER transport vesicles (Figure 3).

As demonstrated in Figure 3, the identification of labeled lipids in ER vesicles justifies the discrepancy in the determined gain of labeled lipids in ER that actively releases transport vesicles to cytosol and thus depletes the radiolabel

\section{Synthesis of Serine-incorporating Lipids in NM}

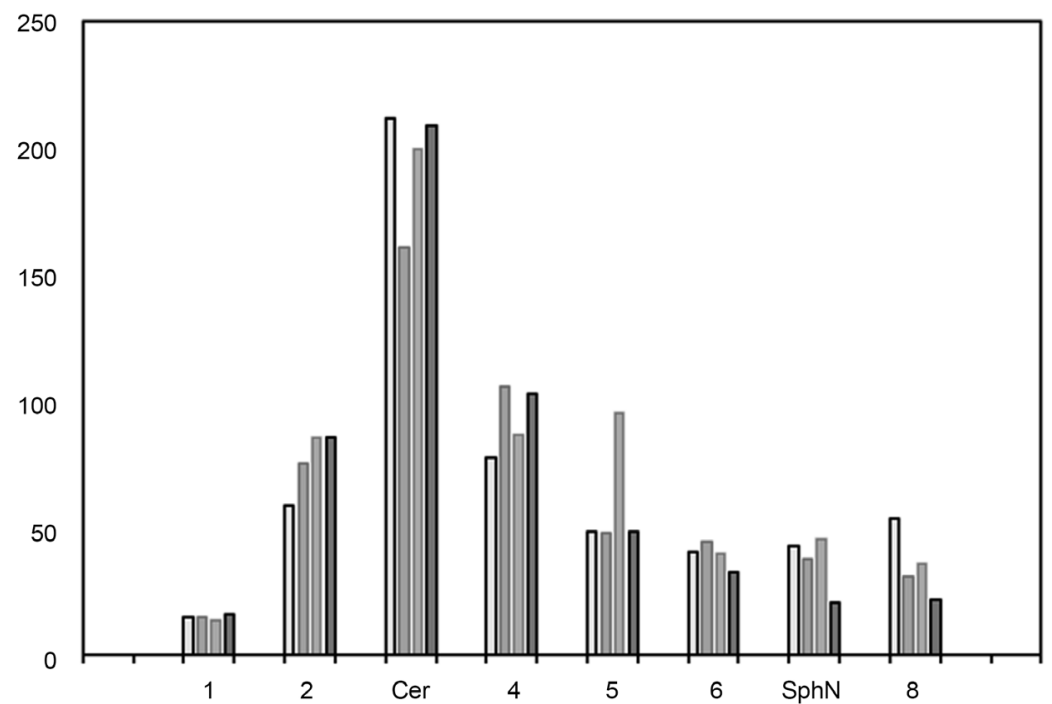

Figure 1. High performance thin layer chromatography (HPTLC) of the serine labeled lipids identified in NM. The chromatography was performed in two solvent systems, one consisting of chloroform/methanol/water $(65: 35: 8, \mathrm{v} / \mathrm{v} / \mathrm{v})$ to separate phosphoglycerides (phosphatidylserine (PS) and its derivatives depicted under area 4, 5, 6, and following alkaline methanolysis treatment in chloroform/methanol/2 $\mathrm{M} \mathrm{NH}_{4} \mathrm{OH}$ to identify ceramides (Cer), sphingosine ( $\mathrm{SphN}$ ) and the unknown products detected in spots 1, 2 and 8, respectively. As shown, the chromatography of the labeled NM lipid extracts was performed on four independent samples. 


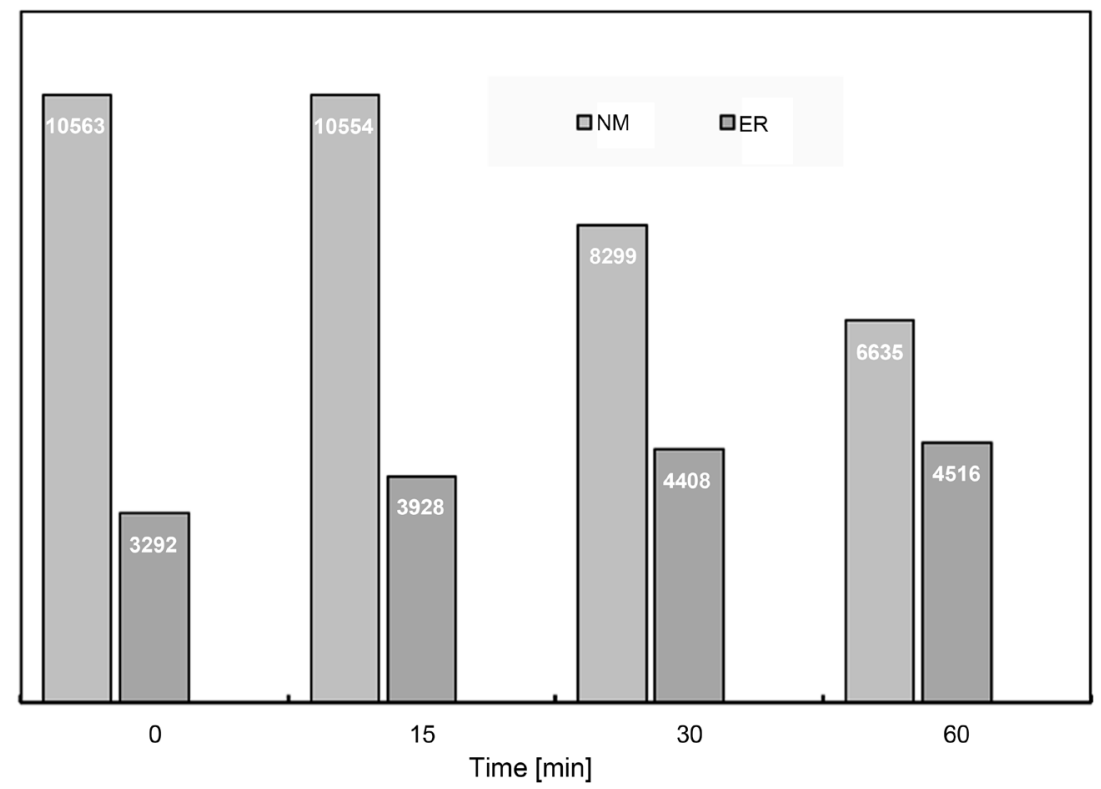

Figure 2. Chase of radiolabeled serine incorporating lipids from NM to ER. As evident from determination of the contents of serine-labeled lipids in samples drawn at 15, 30 and $60 \mathrm{~min}$ the NM labeling was diminishing while ER was gaining the nucleus-exposed membrane. Within $60 \mathrm{~min}$ the nuclear label consisting of $10,563 \mathrm{cpm}$ decreased in the parallel sample to $6635 \mathrm{cpm}$, whereas ER label increased from 3292 to $4516 \mathrm{cpm}$.

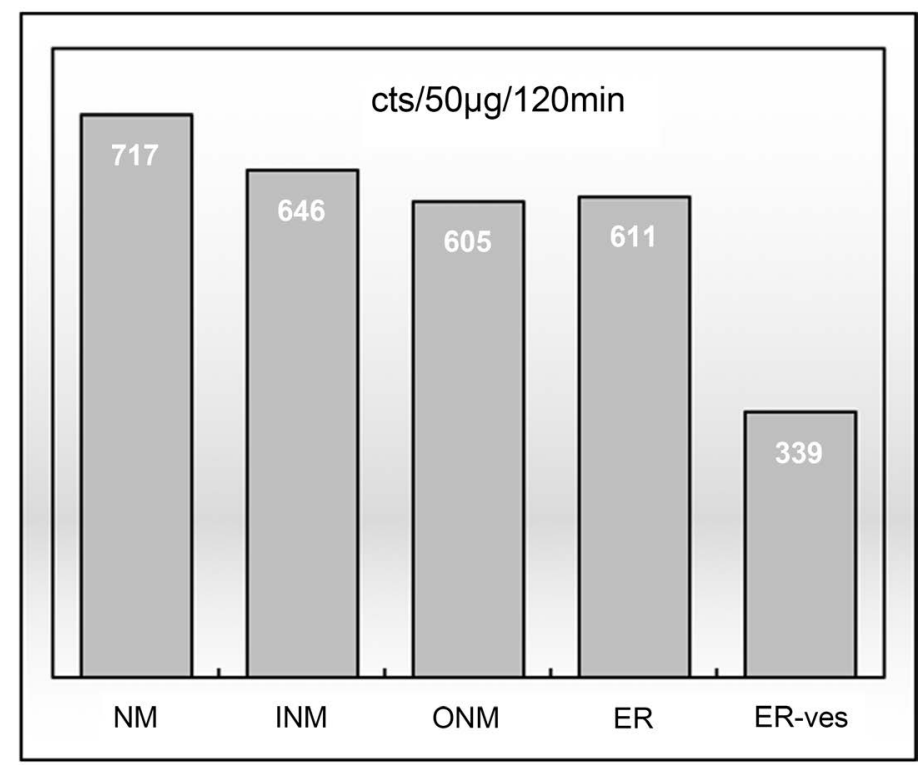

Figure 3. Quantitation of radiolabeled SphN and Cer in the NM, INM, ONM, ER and ER vesicles (ER-ves). The lipid extract derived from $50 \mu \mathrm{g}$ of purified membranes were subjected to alkaline methanolysis to degrade phosphoglycerides depicted in Figure 1 and counted. As shown, the NM, the fraction recognized as INM, ONM, ER and ER-ves contained labeled SphN and Cer products.

transferred from NM to ER. Ideally these experiments should be continued further to account for the nuclear release of radiolabeled ER products for mitochondria, but the technic that allows to quantitate synthesis of sphingolipids 
marking Golgi-dedicated transport, requires degradation of serine-labeled phosphoglycerides and by the same token formation of the Cer-free transport vesicles committed for mitochondria.

Yet, just as appearance of PI in ER [27], these results endorse our concept that the dynamic advance and continuity of NM with ER facilitate progress of the nucleus-designed fragments to ER and produced Cer-labeled Golgi-destined transport vesicles.

The discovery of Cer synthesis in nuclear membranes that along with PI constitute lipid markers of Golgi-destined and ER-assembled transport vesicles reflected on the initiation of the pathway that contributes to synthesis of GSLs and SM in Golgi. As demonstrated by the results shown in Figure 2, the nuclear membranes assemble serine-labeled Cer but question regarding their contribution to growth of the inner or outer leaflet of membrane remain unknown. In contrast to PI, the Cer, to provide lipid core for synthesis of GSLs and SM in Golgi should be incorporated in the inner leaflet of ER and Golgi membranes.

To explore the connection between the Cer synthesis in nuclear membranes and their placement in the specific leaflet of ER and consequently Golgi membranes, the SPT, the enzyme responsible for incorporation of serine label into the SphN of the Cer was investigated. As demonstrated in Figure 4, the SPT activity was found in all membranes derived from nucleus and ER.

As previously demonstrated, the SPT in ER appeared in two molecular forms [29]. The $58 \mathrm{KDa}$ was incorporated into the inner leaflet (IL-ER) whereas 56 $\mathrm{KDa}$ contributed to outer leaflet (OL-ER). This investigation revealed that the enzyme isolated from nuclear membranes was enriched in the $58 \mathrm{KDa}$ form (Figure 5). Based on the previous findings regarding extractability of $56 \mathrm{KDa}$ form with urea, and retention of $58 \mathrm{KDa}$ SPT in ER membranes we have derived with conclusion that inner leaflet of ER, and by the same principle nuclear

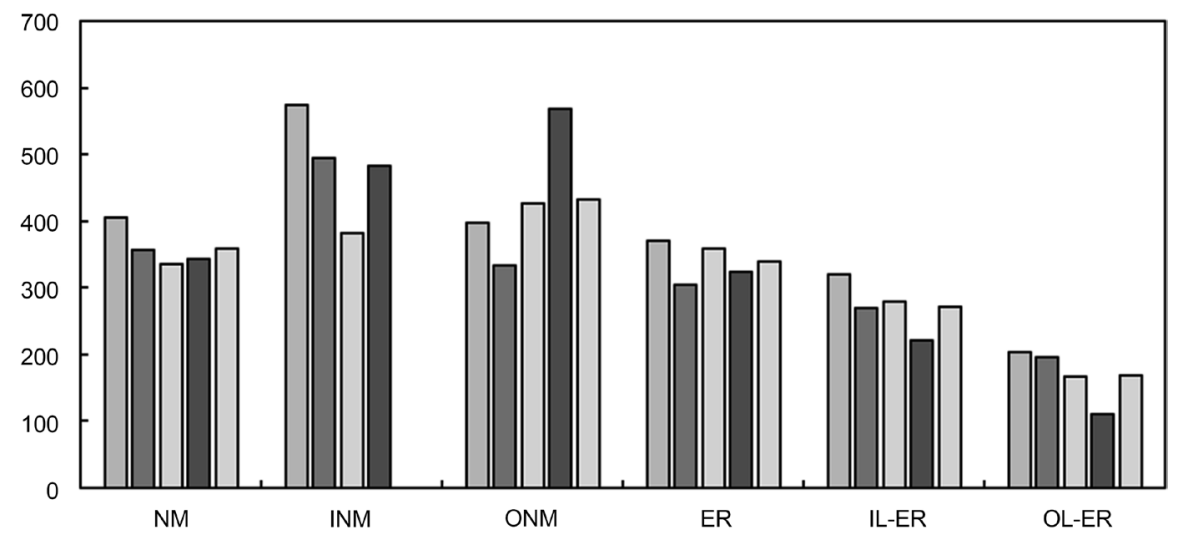

Figure 4. Enzymatic activity of serine palmitoyltransferases (SPT) derived from nuclear and ER membranes. The samples consisting of $50 \mu \mathrm{g}$ of purified membrane protein were used in assay employing radiolabeled serine. Four independent samples of the membrane proteins were utilized. Each bar depicts amount of radiolabel incorporated into SphN in an in vitro SPT assay and reflects counts $/ 120 \mathrm{~min} / \mathrm{sample}$. As demonstrated the NM, its INM and ONM fragments, the ER and its inner and outer leaflet contained active SPT. 


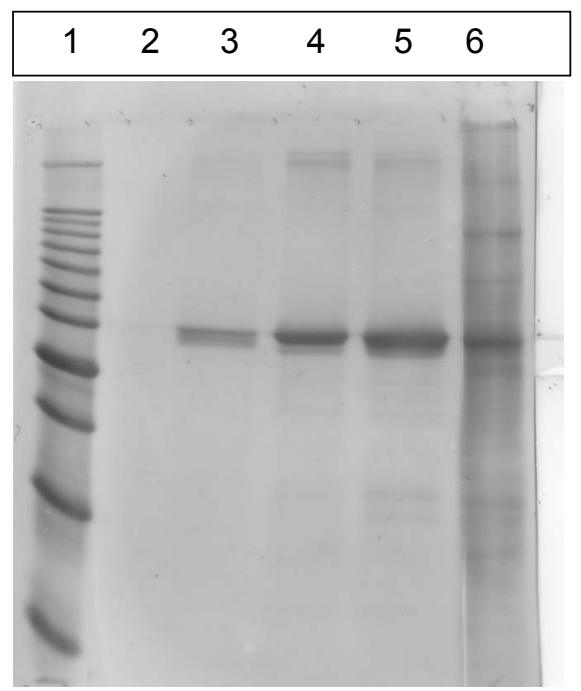

Figure 5. Polyacrylamide gel electrophoresis (PAGE) of the purified (lane 3, 4, 5) and crude (lane 6) SPTs isolated from ER (lane 3), ONM (lane 4) INM (lane 5) and the preparation of NM (lane 6) used for isolation of $58 \mathrm{KDa}$ and generation of the antibodies. The molecular markers are depicted in lane 1 and the sample buffer was introduced in lane 2 .

membranes contain the SPT intercalated into the inner leaflet of the INM and ONM fragments. Hence, the presence of $58 \mathrm{KDa}$ SPT in the inner leaflet of INM and ONM argues for their identity with SPT of the IL-ER and thus provides the support to our concept that nuclear and ER membrane represent continuous membrane whose lipid core and its destination to and from ER is determined by signals entering nucleus. In case of Golgi-dedicated signaling pathway, the presence of Cer core initiated in nuclear membrane and transferred to IL-ER links the process to Golgi synthesis of GSLs and SM that are confined to the inner leaflet of Golgi membrane. In contrast, the Cer core synthesized by the $56 \mathrm{KDa}$ SPT of the OL-ER is involved in synthesis of the SM marking vesicles destined for endosomes and lysosomes [29].

To document the transit of nucleus-designed Golgi-dedicated transport vesicles marked with labeled Cer, the ER-released vesicles (as shown in Figure 3) were subjected to fusion with Golgi and the recovered Golgi membranes analyzed for the presence of Cer and Cer labeled lipids. The fusion experiments of Cer-labeled transport vesicles from ER membranes with Golgi afforded incorporation of the radiolabeled Cer into organelle's GSLs and SM (Figure 6). In turn, the fusion of Golgi-derived transport vesicles with endosomes/lysosomes enriched endosomes with SMase susceptible radiolabeled SM (not shown, but same as demonstrated in [29]), whereas GSL-containing vesicles remained in cytosol [33] [36]. Hence, the fusion experiments utilizing first nucleus initiated Cer-labeled ER vesicles with Golgi, and repeated with Golgi-derived vesicles transferred nucleus-designed membrane through Golgi pathway to cell membranes and to endosome/lysosome organelles. Although the multi-step procedure of fusion and identification of the products is extremely laborious and generates substantial losses, the identification of the Cer labeled GSLs in the 


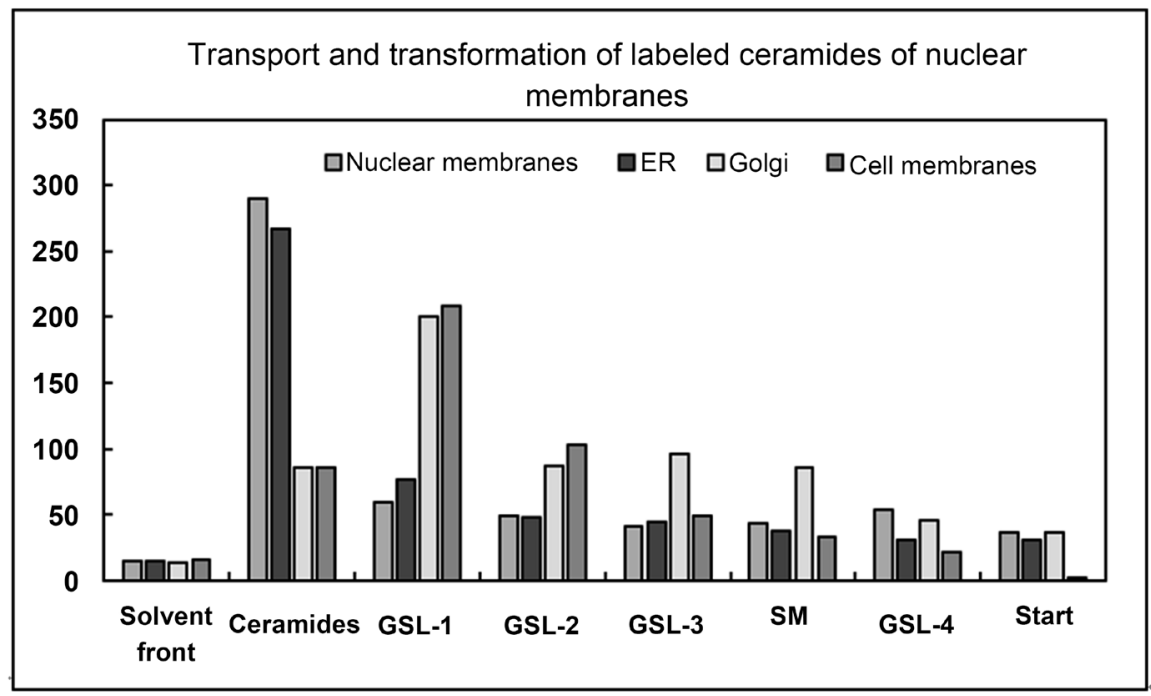

Figure 6. Serine-labeled products revealed in Golgi membrane lipid extract following fusion of ER-transport vesicles containig serine-labeled Cer. The serine-labeled nuclear membrane, ER, and Golgi membranes following fusion with radiolabeled ER-transport vesicles were subjected to lipid extraction, alkaline degradation and HPTLC in sovent system consisting of chloroform/methanol/water (65:35:8, v/v/v) along with Cer, GSLs, and SM standards. Following development and staining, the plates were scored in correspondence to standards, scraped and counted. The same procedure was applied to Golgi derived vesicles incubated with cell membranes.

label-free Golgi demonstrates viability of the system and utilization of the nuclear membranes-derived lipid substrate in the process. The conversion of the Cer to the Cer-labeled GSLs observed in Golgi demonstrates that the presence of the Cer substrate in the inner leaflet of Golgi membrane is crucial for GSL synthesis. Hence, the studies of nuclear membranes, and the results of the investigations utilizing specific lipid marker in form of SphN-labeled Cer allowed us to plot a course that is taken from the nucleus to cell organelles and the membrane, the route that nuclear signals are translated into restitution of the cell compartments and fulfillment of cell-designated function.

The synthetic activity of both SPTs, differing in their extractability from the microsomes and about $2 \mathrm{KDa}$ difference in the molecular weight, was the same whether enzyme was derived from nuclear or ER membranes. On the average, the synthesis of radiolabeled SphN evoked by SPT present in $50 \mu \mathrm{g}$ of nuclear or ER untreated membranes were quite similar (Figure 4) and comparable with those characterized in [29].

However, in further attempt to establish whether difference in molecular weight determine not only intercalation into respective membrane leaflets but also activity of the specific form of SPT, the antibodies against each form were raised and their inhibitory activities determined (Table 1). As demonstrated in the Table the SPT activity of the enzyme in nuclear membranes (NM) was inhibited by $62 \%$ using 58 Abs and $39 \%$ by 56 Abs. When the INM and ONM fragments were assayed independently, both Abs equally inhibited the enzyme activity 
Table 1. Inhibitory activity of the antibodies generated against $58 \mathrm{KDa}$ SPT proteins extracted from ER and $56 \mathrm{KDa}$ from ER SPT released with urea.

\begin{tabular}{cccccccc}
\hline Sample & protein & $\begin{array}{c}\text { protein in } \\
\text { the assay }\end{array}$ & $\begin{array}{c}\text { assay } \\
\text { volume }\end{array}$ & TK buffer & {$\left[{ }^{3} \mathrm{H}\right]$ serine } & Assay vol. & \% inhibition \\
& $\mu \mathrm{g} / \mu \mathrm{l}$ & $\mu \mathrm{g}$ & $\mu \mathrm{l}$ & $\mu \mathrm{l}$ & $\mu \mathrm{l}$ & $\mu \mathrm{l}$ & \\
$\mathrm{NM}+58$ & 3.12 & 50 & 16 & 2.48 & 1.2 & 80 & 62 \\
$\mathrm{NM}+56$ & 3.12 & 50 & 16 & 2.33 & 1.2 & 80 & 39 \\
$\mathrm{INM}+58$ & 3.12 & 50 & 16 & 2.48 & 1.2 & 80 & 52 \\
$\mathrm{INM}+56$ & 3.12 & 50 & 16 & 2.33 & 1.2 & 80 & 41 \\
$\mathrm{ONM}+58$ & 3.12 & 50 & 16 & 2.48 & 1.2 & 80 & 41 \\
$\mathrm{ONM}+56$ & 3.12 & 50 & 16 & 2.33 & 1.2 & 80 & 40 \\
$\mathrm{ER}+58$ & 3.12 & 50 & 16 & 2.48 & 1.2 & 80 & 43 \\
$\mathrm{UE}+58$ & 3.12 & 50 & 16 & 2.48 & 1.2 & 80 & 82 \\
$\mathrm{UE}+56$ & 3.12 & 50 & 16 & 2.33 & 1.2 & 80 & 89 \\
\hline
\end{tabular}

by $40 \%$. The highest, but also equal inhibitory potency of 58 and 56 Abs was observed with the urea released enzyme. On the average, both Abs inhibited the urea-extractible enzyme by $82 \%-89 \%$. It is possible that the displayed differences in the inhibitory activity demonstrated with preparation of membranes, in contrast to solubilized fractions, reflects on accessibility of the membrane integrated SPT. Otherwise, except for the inner or outer leaflet integration, both forms of the enzyme led to formation of Abs with the similar recognition and potency.

The overall similarities in the synthetic activity of SPT and the amount of the product generated from isolated nuclear and ER membranes led us to believe that the purified nuclear and ER samples represent fragments derived from the same membrane that encompases nucleus and ER. If INM and ONM were independent entities, the INM would not contain SPT and generate membrane labeled with SphN and Cer. The presence of SPT and of radiolabeled products in INM indicate that ONM fragment of the membrane continuum advanced from the cytosol-facing, to the segment facing nuclear contents. Such movement allows elaboration of the mebrane that its inner leaflet remains facing ER contents, and consequently the ER generates Golgi-specific vesicles with Cer incorporated into the inner leaflet of their membrane and as such progresses to Golgi. Importently, the membrane continnum asures the uninterrupted contact of nuclear processes with ER and evoke responses that precisely restore cellular organelles and cell membrane.

In conclusion, the significance of our study of Golgi secretory pathway, and findings regarding SPT placement, is in demonstration that cell organelles' membrane lipid composition is determined at the outset of the membrane biogenesis, the process that generates precise, organelle-specific replacement and defines pathway responsible for the precise restitution of the cellular organelles and cell anabolic functions. 


\section{Discussion}

The nuclear outer membrane and ER are studded with ribosomes engaged in the protein renewal of the cell and its organelles [39] [40]. Our investigations on the assembly of the organelles-specific biomembranes reveal that the process is coordinated with simultaneous synthesis and intercalation of lipids that also commences in nuclear membrane and continues throughout ER. The cell membranes designed on nucleus and ER, are revealed in the compartmentalization of the vast array of functions inaugurated in nucleus, and fulfilled by the cell organelles and cell membrane. On one facet the nuclear membrane gates multiplicity of highly coordinated processes that regulate nuclear mechanics and gene expression and provides strict platform for the recognition and transport of substances between nucleoplasm and cytoplasm, while on the opposing surface, it provides binding sites for the released to cytosol ribosomes that translate explicit mRNAs and faithfully recreate cell-specific proteins [39] [40]. Thus, the membrane barrier biogenesis and its components directly respond to the nuclear processes evoked by signaling. The transcribed messages revealed in the formation of the specific nuclear membrane segments meant for repair of multiple organelles, upon receiving suitable cargo in ER, undergo vesicles formation, fission from ER and journey to their outer membrane signal-pointed site [27] [28] [29] [30]. As determined in earlier investigations of the composition of ER-derived transport vesicles, the responses are transcribed into organelle specific combination of proteins and membrane lipids, and hence imply that each segment of the organelle specific membrane is translated by cluster of ribosomes dedicated to the task [39] [40]. Since ribosomes are only attached to so called outer nuclear membrane (ONM), the question arose as to the biosynthesis of the inner nuclear membrane (INM), how the INM is remodeled upon signal-initiated processes, and how the translates of the ribosomes reach the inaccessible membrane.

In the orthodox view of nuclear membrane, the nucleus-ER structural assembly explains ONM responsiveness to cytosol-promoted signals but is isolated from specific nuclear triggers that initiate the specific responses embodied in formation of the membranes required by the cell. By applying same mainstream concept, the INM is the entity isolated from ribosome-containing ONM and ER, and thence the synthetic processes on ONM determined by ribosomes and mRNA delivered to cytosol are not building and incorporating signal-demanded changes in INM.

While studying conversions of nuclear membrane phosphoinositides, our investigative results provided strong support to the concept that nuclei are embraced not by double but by single bilayer membrane that is an uninterrupted continuum protracted into formation recognized as ER. The unconventional concept of the nuclear barrier induced by the data acquired on nuclear phosphatidylinositol (PI) synthesis [27], provided realistic explanation of the signal-prompted changes in INM and ONM. If, on the other hand, the INM and ONM represented two independent membranes enticing nuclear content, the PI 
products would be retained in INM.

The further support to our argument of the uninterrupted membrane continuum embracing nucleus and ER emerged from the investigation of nuclear membranes involvement in the SphN and Cer synthesis which along with PI represent specific markers of the ER-derived transport vesicles dedicated to Golgi pathway [29] [32] [33] [35]. Continuing with our counterargument against independent ONM and INM entities, we challenge that if so, the SPT enzyme generating SphN and Cer-containing lipids would not be detectable in INM. Moreover, the Cer which are exclusively incorporated during biomembranes synthesis in response to cellular demand for assembly of ER transport vesicles destined for Golgi would only be found in the serine-labeled newly synthesized lipid components of ONM. The results of the investigation on serine-labeled lipids synthesis in NM have uncovered that the Cer lipids and active SPT enzyme, are present in NM, and the INM and ONM segments. These discoveries demonstrated and further supported our stand that nuclear barrier is built as uninterrupted single membrane continuum that responds to nucleus and cytosol-delivered signals materialized in membrane synthesis. Such process, impose membrane movement from the cytosol-facing portion recognized as ONM to nuclei-exposed putative INM and back to cytosol exposed ER.

As the studies on transport vesicles destined for Golgi pathway determined, PI is incorporated into the outer leaflet of the membrane [27]. Indeed, its presence in the cytosol facing leaflet is in good agreement with destination-specific modification of PI arriving to Golgi and undergoing cytosol-triggered transformation into PI3P and PI4P [29] [36]. Thus, as determined in our earlier investigations [36], the transport vesicles outfitted with PI3P marker and cytosol-activated PI3K find their final destination by fusing with apical epithelial membrane, whereas portion with PI4P displays affinity for basolateral cell membrane and endosomes [29]. At that stage of our investigations, the directional specificity of transport vesicles was assigned to the nuclear membrane embedded PI and PIK that committed them to reach exact final destination. Moreover, it demonstrated that PIs in the assembled membranes and in the direct contact with cytosol do not require the transfer (flipping) of lipids from one facet to another. Similarly, the studies described in this paper reveal positional significance of the Cer intercalation in the outer and inner leaflet of the membrane. Without flipping, these Cer serve in the inner leaflet as lipid cores for the synthesis of GSLs and SM and, after vesicles cargo delivery to cell membrane (basolateral release of the albumin and/or apical release of glycoprotein), face correctly the outer environment of the cell, or if intercalated into outer leaflet of the vesicles are committed for endosomes and lysosomes [29] [31]. The examination of the SphN-containing nuclear products delivered to Golgi via ER transport vesicles and transformed in Golgi into GSLs and SM demonstrated that the composition of the inner leaflet of ER transport vesicles is achieved through nuclear presence of 58KDa SPT and, incorporation of its SphN into Cer products to the inner leaflet of the mem- 
brane, determines the synthesis of GSL and SM dedicated for cell membrane restitution and fulfillment of its secretory function.

Collectively, the evidence presented allows to conclude that from the outset the nucleus-initiated and ER completed transport vesicles differ in their lipid composition in the outer and inner leaflet, and together with the integral membrane proteins, their pathway for terminal destination is demarcated.

Without a doubt, the attributes of the ER-generated transport vesicles reflect nuclear release of the clusters of ribosomes and mRNA that elaborate specific sculpt of cell membranes [39] [41] [42]. The fact that SPT enzyme and that its products were identified in nuclear membranes and further traced in the inner leaflet of ER and Golgi defines the membrane movement from nuclear membranes, through ER and its vesicular products to Golgi and then to cell apical or basolateral membrane.

Based on the quantitative comparison of SPT presence in the nuclear membranes of the secretory epithelial cells it is tempting to suggest that the expression of the $58 \mathrm{KDa}$ SPT enriched in nuclear portion reflects on the degree of the epithelial cells restitution and apical/basolateral transport. Also, but in contrast, it is possible that $56 \mathrm{KDa}$ SPT associated with ER outer leaflet and, involved in synthesis of SM in the outer leaflet of endosome-directed transport vesicles, is expressed in larger quantity on the account of drug-induced cellular degradation that demands transport of the proteolytic enzymes to lysosomes [31]. By the extension, it is quite possible that the final destination markers for Golgi vesicles pathway integrated into the membrane at the stage of nuclear biomembrane assembly is quintessential in synthesis of the organelle-specific cargo in ER and its delivery to the exact site. This presumptive argument requires further exploration of other protein markers that accompany SPT in the vesicles containing SM and transporting cargo to basolateral epithelial segment of the cell membrane or endosomes and lysosomes [39] [40], and all is decided by the cluster of ribosomes that are simultaneously involved in the synthesis of the signal-demanded membranes and secretory products [41] [42].

In the initial early investigations that elucidated starting points for the intracellular transport we had not enough background to embrace the concept that specific lipids may dictate destinations of the transport vesicles to Golgi or mitochondria, or how the organelles renewed their structures and vitality [26] [32] [33] [34] [35]. However, the later concentration on role of lipids in the demarcation of the restoration pathways of cell organelles allowed us to draw a highly specific and coherent picture demonstrating that specificity of the nuclear products precisely determines vesicles destinations. The cellular events followed through lipids synthesis dictated by nucleus receiving signals translated into ER-assembled products map the path to either Golgi or mitochondria [28] [30]. As recently determined, the mitochondrial pathway is defined by the presence of phosphatidylglycerol (PG), the proteins that restore outer mitochondrial membrane, intramitochondrial delivery of the enzymes that generate cardiolipin, and 
the absence of PI and Cer in the mitochondria-destined vesicles [28] [30]. Again, entire process is highly refined by combination of the specific proteins and lipids that are interwoven into the specific membrane required for the specific site of the organelle renewal and restoration of its optimal function [32] [35] [39] [40]. The outcome of both, Golgi and mitochondria pathways, is strictly controlled by the cell native cytosol, since fusion of the ER-released vesicles with the accepting organelle depends on the cytosolic presence of the organelle-specific phospholipases [30] [31] [36]. Hence, utilization of the cytosol derived from the cultured cells, different tissue [43], or from animals subjected to drug treatment [31], generate results reflecting impact of foreign cytosol, or the drug, on gene expression in the particular cells including cytosol-released proteins.

The dependence of the intracellular transport on cytosolic milieu was not considered in earlier investigations that relied on various fraction of proteins isolated from brain tissue and might as well be phospholipases depleted [43] [44] [45]. While this paradigm became the beacon for future investigations, the studies drifted to protein-only complexes and were not able to provide reasonable explanation for multiplicity of the pathways initiated by posttranslational changes in transport vesicles determining their final destination. The stand in the general assumption that any mixture of lipids provides sufficient environment to demonstrate protein function and specificity reflects imprecise reasoning and demands investigations that include the input of gene expression on the early stages of membrane assembly by following synchronized synthesis and intercalation of protein and lipids into ER that is later revealed in the membranes of the organelles or cells [39] [40] [41] [42].

Collectively, our study of membrane lipids of the Golgi and mitochondrial vesicular pathway with specific involvement of innate cytosol, generate picture reflecting extremely ordered cellular activity that only then is capable to restore entire structure without errors in the assembly or function. From our point of view, the results from the commonly accepted investigative schemes employing individual components freed from confines of cell organelles do not capture true cellular order [39]. Also, the freedom of protein interaction released from the shattered (lysed) organelles generates convolutedness of the processes that do not take place in an undisturbed cell structure. The lysis-created cocktail of effects generates skewed and distorted view conflicting the true in vivo and in situ observed consequences of cellular signaling. As to the prevailing view of the existence of double and independent nuclear membranes separated by perinuclear space, the concept does not explain functional link of the inner nuclear membrane segments with cytosol. In our understanding of the intracellular membranes properties, and as our investigation suggest the segments recognized as INM and ONM constitute portions of the one continuum that has been interrupted during preparation of the nuclei and ER and as ER structures referred to as ER microsomes should be recognized as nuclear microsomes. The NM involvement in the anabolic-synthetic movement reflected in appearance of lipids 
generated through action of its membrane intercalated SPT provides evidence that INM is not physically separated from ONM and ER, but suggest that the subpool of mRNA translated by the ribosomes attached to nuclear microsomes is involved in restitution of the inner leaflet of the nuclear-ER membrane [40]. In our studies, this is evident in the presence of $58 \mathrm{kDa}$ SPT and its products that are essential for the synthesis of GSLs and SM in the inner leaflet of Golgi membrane.

The processes characterized by the investigations of ribosome footprints [39], heterogeneous ribosome distinct subpools [40] and the presence of the specific SPT on the inner and outer leaflet of ER imply that the placement of the specific lipids and protein is determined during early assembly of the membrane. The composition of the membrane and delivery of the synthetic cargo to the appropriate organelle are not relying on the process of flipping and flippases [46]. Contrary to common conjecture on the mechanisms of intracellular protein transport and protein involved in the intracellular lipid transport [16] [18] [47], our investigations reveal that membrane proteins and lipids of cell and its organelles are defined on the nucleus facing membrane. As much as is known about the principle processes of protein synthesis, the driving force for the genome translation, and nuclear envelope as a "signaling node" in the development and disease [11], our findings add to the manifold complexity of ER-nuclear barrier membrane and decipher further the complexity of the cellular organization. The reality of the process is manifested in synthesis of transport vesicles initiated by nucleus-induced steps that coordinately intercalate specific proteins and lipids into membrane, that are completed in membrane extension commonly recognized as ER, and tailored to deliver cargo and replenish organelle of the specific pathway.

A new concept on the flow of information from nucleus to cell specific entities developed from the investigative findings dedicated to cell membranes synthesis, illustrated through identification of the specific lipids defining organelle membrane, allowed us to decipher cellular pathways that are defined in nuclear membrane by the simultaneous intercalation of the membrane proteins and lipids, the process that guards cellular structure from alterations, provides precise and timely repair and restitution, and retains the cell's unmodified function.

\section{References}

[1] Burke, B. and Ellenberg, J. (2002) Remodeling the Walls of the Nucleus. Nature Reviews of Molecular Cell Biology, 3, 487-497. https://doi.org/10.1038/nrm860

[2] Hetzer, M.W., Walther, T.C. and Mattaj, I.W. (2005) Pushing the Envelope: Structure, Function, and Dynamics of the Nuclear Periphery. Annual Review Cell Development Biology, 21, 347-380. https://doi.org/10.1146/annurev.cellbio.21.090704.151152

[3] D’Angelo, M.A. and Hetzer, M.W. (2005) The Role of the Nuclear Envelope in Cellular Organization. Cell Molecular Life Sciences, 63, 316-332. https://doi.org/10.1007/s00018-005-5361-3 
[4] Skinner, B.M. and Johnson, E.E.P. (2017) Nuclear Morphologies: Their Diversity and Functional Relevance. Chromosoma, 126, 195-212. https://doi.org/10.1007/s00412-016-0614-5

[5] Webster, M., Witkin, K.L. and Cohen-Fix, O. (2009) Sizing up the Nucleus: Nuclear Shape, Size and Nuclear-Envelope Assembly. Journal Cell Sciences, 122, 1477-1486. https://doi.org/10.1242/jcs.037333

[6] Pueschel, R., Coraggio, F. and Meister, P. (2016) From Single Genes to Entire Genomes: The Function of Nuclear Organization. Development, 143, 910-923. https://doi.org/10.1242/dev.129007

[7] Lusk, C.P., Blobel, G. and King, M.C. (2007) Highway to the Inner Nuclear Membrane. Nature Reviews Molecular Cell Biology, 8, 414-420. https://doi.org/10.1038/nrm2165

[8] Siniossoglou, S. (2009) Lipins, Lipids and Nuclear Envelope Structure. Traffic, 10, 1181-1187. https://doi.org/10.1111/j.1600-0854.2009.00923.x

[9] Irvine, R.F. (2003) Nuclear Lipid Signalling. Nature Reviews Molecular Cell Biology, 4, 349-360. https://doi.org/10.1038/nrm1100

[10] Stewart, C.L., Roux, K. and Burke, B. (2007) Blurring the Boundary: The Nuclear Envelope Extends Its Reach. Science, 318, 1408-1412. https://doi.org/10.1126/science.1142034

[11] Dauer, W.T. and Worman, H.J. (2009) The Nuclear Envelope as a Signaling Node in the Development and Disease. Developmental Cell, 17, 626-638. https://doi.org/10.1016/j.devcel.2009.10.016

[12] Jaffarelli, M.H., Vijayaraghaven, B., Figueroa, R.A., Crafoord, E., Gudise, S., Larsson, V.J. and Hallberg, E. (2014) MCLIP, an Effective Method to Detect Interactions of Transmembrane Proteins of the Nuclear Envelope in Live Cells. Biochimica et Biophysica Acta, 1838, 2399-2403.

[13] Amlacher, S., Sarges, P., Flemming, D., van Noort, V., Kunze, R., Devos, D.P., Arumugam, M., Bork, P. and Hurt, E. (2011) Insight into Structure and Assembly of the Nuclear Pore Complex by Utilizing the Genome of Eukaryotic Thermophile. Cell, 146, 277-289.

[14] Korfali, N., Wilkie, G.S., Swanson, S.K., Srsen, J., de Las Heras, J., Batrakou, D.G., Malik, P., Zuleger, N., Kerr, A.R., Florens, L. and Schirmer, E.C. (2012) The Nuclear Proteome Differs Notably between Tissues. Nucleus, 3, 552-564.

[15] Faini, M., Beck, R., Wieland, F.A.T. and Briggs, J.A.G. (2013) Vesicles Coats: Structure, Function, and General Principle of Assembly. Trends in Cell Biology, 23, 279-288. https://doi.org/10.1016/j.tcb.2013.01.005

[16] Whitelegge, J. (2011) Up-Close with Membrane Lipid Complexes. Science, 334, 320-321. https://doi.org/10.1126/science.1214084

[17] Buszczak, M., Signer, R.A.J. and Morrisons, J. (2014) Cellular Differences in Protein Synthesis Regulate Tissue Homeostasis. Cell, 159, 242-251. https://doi.org/10.1016/j.cell.2014.09.016

[18] Mellman, I. and Nelson, W.J. (2009) Coordinated Protein Sorting, Targeting and Distribution in Polarized Cells. National Review of the Molecular Biology, 9, 833-845. https://doi.org/10.1038/nrm2525

[19] van Meer, G. (1998) Lipids of the Golgi Membrane. Trends in Cell Biology, 8, 29-33. https://doi.org/10.1016/S0962-8924(97)01196-3

[20] Ladinsky, M.S., Mastronarde, D.N. and McIntosh, J.R. (1999) Golgi Structure in Three Dimensions: Functional Insights from the Normal Kidney. Journal of Cell 
Biology, 144, 887-901. https://doi.org/10.1083/jcb.144.6.1135

[21] Lingwood, D. and Simon, K. (2010) Lipid Rafts as a Membrane Organizing Principle. Science, 327, 46-50. https://doi.org/10.1126/science.1174621

[22] Bogdanov, M., Dowhan, W. and Vitrac, H. (2014) Lipids and Topological Rules Governing Membrane Protein Assembly. Biochimica at Biophysica Acta, 1843, 1475-1488. https://doi.org/10.1016/j.bbamcr.2013.12.007

[23] Isaksson, S., Watkins, E.B., Browning, K.L., Lind, T. K., Cárdenas, M., Hedfalk, K., Höök, F. and Andersson, M. (2017) Protein-Containing Lipid Bilayers Intercalated with Size-Matched Mesoporous Silica Thin Films. NANO Letters, 17, 476-485. https://doi.org/10.1021/acs.nanolett.6b04493

[24] Eeman, M. and Deleu, M. (2010) From Biological Membranes to Biomimetic Model Membranes. Biotechnology, Agronomy, Society and Environment, 14, 719-736.

[25] Gruenbaum, Y. and Foisner, R. (2015) Lamins: Nuclear Intermediate Filament Proteins with Fundamental Functions in Nuclear Mechanics and Genome Regulation. Annual Review of Biochemistry, 84, 131-164.

https://doi.org/10.1146/annurev-biochem-060614-034115

[26] Slomiany, A. and Slomiany, B.L. (2010) Cell Membranes Composition Is Defined in ER and Their Restitution Proceed by en bloc Fusion of ER Generated Transport Vesicles. Health, 2, 1444-1454. https://doi.org/10.4236/health.2010.212214

[27] Slomiany, A. and Slomiany, B.L. (2011) Transformation of Phosphatidylinositol Phosphates in the Outer and Inner Nuclear Membrane Are Linked to Synthesis and Restitution of Cellular Membranes. Health, 3, 187-199. https://doi.org/10.4236/health.2011.34035

[28] Slomiany, A. and Slomiany, B.L. (2012) Phosphatidylglycerol-Containing ER Transport Vesicles Built and Restore Outer Mitochondrial Membrane and Deliver Nuclear DNA Translation Products to Generate Cardiolipin in the Inner Mitochondrial Membrane. Advances in Biological Chemistry, 2, 132-145. https://doi.org/10.4236/abc.2012.22016

[29] Slomiany, A. and Slomiany, B.L. (2014) Concluding Step in Cell Restitution Cycle: ER Transport Vesicles with Sphingolipids in the Outer Leaflet of the Membrane Restore Lysosomes. Advances in Biological Chemistry, 4, 301-327. https://doi.org/10.4236/abc.2014.45036

[30] Slomiany, A. and Slomiany, B.L. (2017) Mitochondrial Membranes Restitution Proceeds via Vesicular Import from ER and Cytosol. Counterparts' Resemblances and Variances in Mitochondria and Golgi Pathways. Advances in Biological Chemistry, 7, 1-26. https://doi.org/10.4236/abc.2017.71001

[31] Slomiany, A. and Slomiany, B.L. (2015) Drug-Induced Disparities in Cell Restoration and Debridement: Ethanol-Triggered Nuclear Misreading of the Restitution Cues. Global Journal of Medical Research. Drug Discovery, Toxicology and Medicine, 15, 22-37.

[32] Slomiany, A., Grzelinska, E., Grabska, M., Morita, M. and Slomiany, B.L. (1992) Intracellular Processes Associated with Glycoprotein Transport and Processing. Archives of Biochemistry and Biophysics, 298, 167-175.

[33] Slomiany, A., Grabska, M., Piotrowski, E. and Slomiany, B.L. (1994) Intracellular Processes Associated with Vesicular Transport from Endoplasmic Reticulum to Golgi and Exocytosis. Archives of Biochemistry and Biophysics, 310, 247-255. https://doi.org/10.1006/abbi.1994.1164

[34] Slomiany, A., Grabska, M. and Slomiany, B.L. (2006) Homeostatic Restitution of Cell Membranes. Nuclear Membrane Lipid Biogenesis and Transport of Protein 
from Cytosol to Intranuclear Spaces. International Journal of Biological Sciences, 2, 216-226. https://doi.org/10.7150/ijbs.2.216

[35] Slomiany, A., Sano, S., Grabska, M., Yamaki, K. and Slomiany, B.L. (2004) Gastric Mucosal Cell Homeostatic Physiome. Critical Role of ER-Initiated Membrane Restitution in the Fidelity of Cell Function Renewal. Journal of Physiology and Pharmacology, 55, 837-860.

[36] Slomiany, A., Nowak, P., Piotrowski, E. and Slomiany, B.L. (1998) Effect of Ethanol on Intracellular Vesicular Transport from Golgi to the Apical Membrane. Role of Phosphatidyl 3-Kinse and Phospholipase $\mathrm{A}_{2}$ in Golgi Transport Vesicles Association and Fusion with Apical Membrane. Alcohol, Clinical and Experimental Research, 22, 167-175.

[37] Houtkooper, R.H. and Vaz, F.M. (2008) Cardiolipin, the Heart of Mitochondrial Metabolism. Cellular and Molecular Life Sciences, 65, 2493-2206.

https://doi.org/10.1007/s00018-008-8030-5

[38] Scarpulla, R.C. (2008) Transcriptional Paradigms in Mammalian Biogenesis and Function. Physiological Review, 88, 611-638.

https://doi.org/10.1152/physrev.00025.2007

[39] Ingolia, N.T. (2016) Ribosome Footprint Profiling of Translation throughout the Genome. Cell, 165, 22-33. https://doi.org/10.1016/j.cell.2016.02.066

[40] Shi, Z., Fujii, K., Kovary, K.M., Genuth, N.R., Röst, H.L., Teruel, M.N. and Barna, M. (2017) Heterogeneous Ribosomes Preferentially Translate Distinct Subpools of mRNAs Genome-Wide. Molecular Cell, 67, 71-83.

https://doi.org/10.1016/j.molcel.2017.05.021

[41] Sonenenberg, N. and Hinnebusch, A.G. (2009) Regulation of Translation Initiation in Eukaryotes: Mechanism and Biological Targets. Cell, 136, 731-745. https://doi.org/10.1016/j.cell.2009.01.042

[42] Reid, D.W. and Nicchitta, C.V. (2012) Primary Role for Endoplasmic Reticulum-Bound Ribosomes in Cellular Translation Identified by Ribosome Profiling. Journal Biological Chemistry, 287, 5518-5527. https://doi.org/10.1074/jbc.M111.312280

[43] Rothman, J.E. (1994) Mechanisms of Intracellular Protein Transport. Nature, 372, 55-63. https://doi.org/10.1038/372055a0

[44] Sudhof, T.C. and Rothman, J.E. (2009) Membrane Fusion: Grappling with SNARE and SM Proteins. Science, 323, 474-477. https://doi.org/10.1126/science.1161748

[45] Glick, B.S. and Nakano, A. (2009) Membrane Traffic within the Golgi Apparatus. Annual Review of Cell Development Biology, 25, 113-132. https://doi.org/10.1146/annurev.cellbio.24.110707.175421

[46] Van Meer, G., Halter, D., Sprong, H., Somerharju, P. and Egmond, M.R. (2006) ABC of Lipid Transporters: Extruders, Flippases, or Flopless Activators? FEBS Letters, 580, 1171-1177.

[47] Lemmon, M.A. (2008) Membrane Recognition by Phospholipids Binding Proteins. National Review of the Molecular Cell Biology, 9, 99-111.

https://doi.org/10.1038/nrm2328 